\title{
La cité et ses images
}

La collection iconographique de Marcel Poëte : extraits

\section{Steven Melemis}

\section{(2) OpenEdition}

Journals

Édition électronique

URL : http://journals.openedition.org/crau/487

DOI : $10.4000 /$ crau. 487

ISSN : 2547-5746

Éditeur

Éditions du patrimoine

Édition imprimée

Date de publication : 1 mars 2014

Pagination : 85-106

ISBN : 978-2-7577-0359-5

ISSN : 1296-4077

Référence électronique

Steven Melemis, "La cité et ses images », Les Cahiers de la recherche architecturale et urbaine [En ligne], 29 | 2014, mis en ligne le 13 septembre 2017, consulté le 19 avril 2019. URL : http://

journals.openedition.org/crau/487; DOI : 10.4000/crau.487 


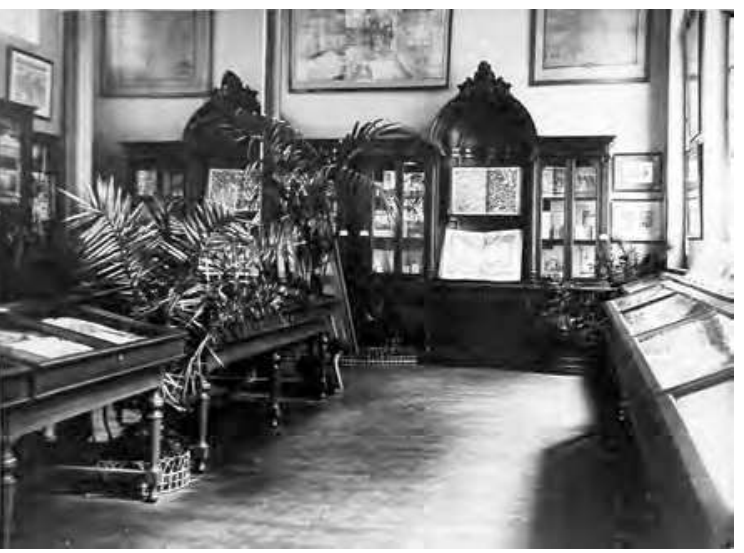

Exposition « La vie populaire à travers l'image et l'illustration » de 1907. Photographie 1907.

Collection photographique de la BHVP. D.R.

* Architecte, enseignant à l'Énsa de Paris-Malaquais (responsable du département "Architecture, villes, territoires"), membre associé au laboratoire IPRAUS (UMR AUSser 3329), membre du collectif pluridisciplinaire "Bazar urbain ». II prépare une thèse sur Marcel Poëte et sa collection iconographique, sous la direction de Jean-Louis Cohen et Yannis Tsiomis.

\section{Présentation par Steven Melemis*}

Derrière le personnage se cachait un objet, d'un grand intérêt. Le personnage en question, Marcel Poëte - archiviste, historien, urbaniste, éducateur et vulgarisateur de première heure dans le champ de l'urbanisme en France, est une figure connue de l'histoire d'urbanisme, grâce à des travaux biographiques récents ${ }^{1}$ mais aussi par certains de ses ouvrages: I'Introduction à l'urbanisme $(1929)^{2}$, Une vie de cité. Paris de sa naissance à nos jours (1924-1931) ${ }^{3}$, Aperçu historique, tome I. Rapports de la Commission d'extension de Paris de $1913^{4}$. Quant à l'objet, il évolue au sein d'une configuration de forces vives à l'œuvre dans la vie publique à Paris pendant les trente premières années du siècle dernier, c'est-à-dire à l'époque des premières réflexions sur la capitale à l'échelle de ce que nous appelons aujourd'hui le Grand Paris. Il ouvre d'intéressantes perspectives de réflexion (notamment) pour I'histoire intellectuelle et culturelle de l'urbanisme. L'objet en question est une archive photographique d'iconographie urbaine comprenant pour l'essentiel des vues de Paris, archive qui a été entamée pendant l'année 1907 et dont les dernières pièces datent d'un moment indéterminé dans les années $1920^{5}$. Depuis au moins une cinquantaine d'années, elle est restée enfermée
1. Voir Donatella Calabi, Marcel Poëte et le Paris des années vingt : aux origines de l'histoire des villes. Paris, L'Harmattan, 1997 (trad.fr. Pierre Savy). Voir également les éléments biographiques présentés par Hubert Tonka pour la réédition de I'Introduction à I'urbanisme, Paris, Sens et Tonka, 2000.
3. M. Poëte, Une vie de cité. Paris de sa naissance à nos jours, 4 volumes, Paris, Picard 1924-1931 : La jeunesse. Des origines aux temps modernes ; La cité de la Renaissance; La spiritualité de la cité classique. Les origines de la cité moderne; Album.

4. M. Poëte, Louis Bonnier, Rapports de la Commission d'extension de Paris : I. Aperçu historique; Il. Considérations préliminaires. La circulation et les espaces libres, Paris, Imprimerie Chaix, 1913. 
dans les réserves de la Bibliothèque historique de la Ville de Paris (BHVP), le lieu où cette collection a été créée à l'instigation de Marcel Poëte alors bibliothécaire en chef de l'institution'. À ses débuts, la collection se constitue grâce à un travail de dépouillement (commencé en 19067) diversifié, exhaustif et aux proportions colossales de sources de l'histoire de Paris repérées dans tous les dépôts publics de Paris, notamment aux Archives nationales, à la Bibliothèque nationale, à I'hôtel Carnavalet et à la BHVP, Le recueil iconographique s'amplifiera dès 1908 , en intégrant de nombreuses contributions de collectionneurs privés. La collection impressionne d'abord simplement par son ampleur: un peu plus de 18000 clichés de projection présentant des vues reproduites photographiquement selon un processus d'impression de gélatine sur verre. Photographiées avec soin, d'une grande précision, elles sont encadrées de métal noir en format $8 \times 10 \mathrm{~cm}$, rognées avec un film adhésif noir, numérotées, marquées d'un titre et

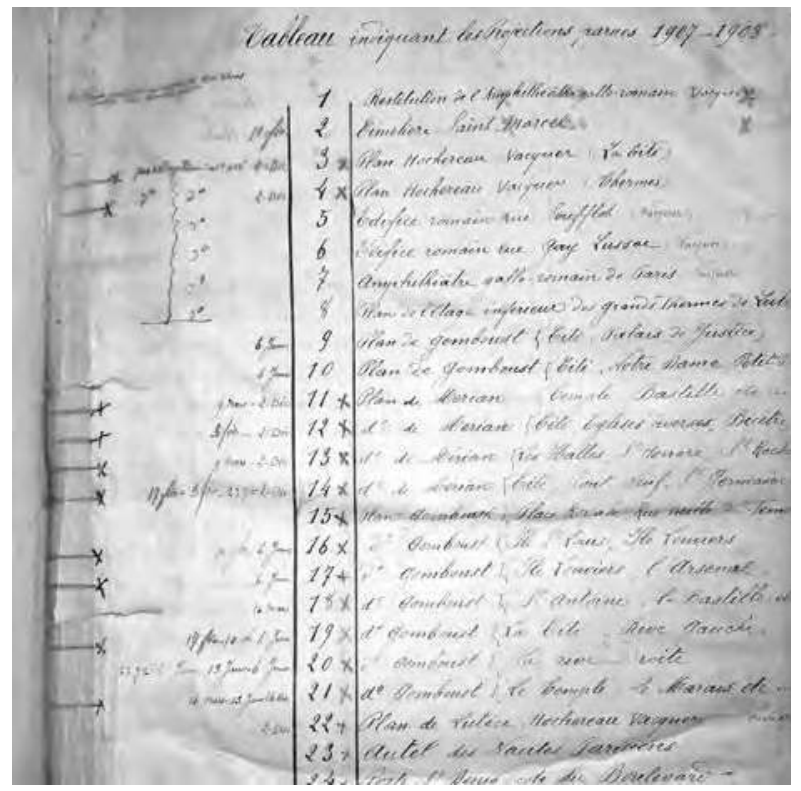

Liste de projections, vol. 1 (extrait de la première page). Document non classé. rangées dans à peu près une centaine de tiroirs. Un certain nombre d'entre elles sont coloriées au pinceau. Un fichier de cartes, comprenant 24 tiroirs, contient un système de classification topographique et thématique; la plupart de ces cartes comportent une reproduction photographique d'un ou de plusieurs clichés ainsi que des annotations diverses faites à la main. Par ailleurs, grâce aux deux premiers volumes d'une Liste de projections pour la collection retrouvés récemment à la BHVP (un troisième aurait été détruit accidentellement), nous possédons un inventaire d'un peu moins de deux tiers des projections, numérotées par ordre d'entrée et couvrant la période de 1907 à 1918. Ces deux volumes comprennent des indications sur les vues conservées, leurs intitulés et souvent également leurs sources, les dates d'acquisition et des indications concernant leur usage au moment d'être intégré à la collection. Enfin, dans les Papiers Poëte conservés à la BHVP, on trouve de nombreuses listes de projections faites à l'occasion de cours et de conférences.

Bien plus que par son importance ou son organisation finalement assez conventionnelle, le regroupement de vues étonne par la richesse et la diversité des représentations visuelles qu'il recèle des espaces urbains parisiens, ainsi que de la population parisienne, essentiellement depuis
5. En effet rien n'indique les dates d'acquisition des derniers clichés de la collection et nous ne disposons pas de liste de projections pour la période après 1913.

6, Dès cette date, il dirige également les Travaux historiques de la Ville de Paris et il est Secrétaire la Commission du Vieux Paris.

7. La collection de notices descriptives écrites de l'Office de bibliographie parisienne qui est issue de cette collecte se retrouve à I'Institut de topographie parisienne des Archives nationales. Le recueil a également donné lieu à un volume imprimé de sources de l'histoire de Paris. Quant à la collection iconographique, sa classification n'a jamais été publiée ou rendue publique. 
le $\mathrm{xv}^{\mathrm{e}}$ siècle et jusqu'au moment de sa création. En effet, au moins 15000 de ces clichés reproduisent des estampes et imprimés de toutes sortes, dessins, pièces typographiées et photographies, en entier ou en détail. À partir de 1919 environ, la collection intégrera également des cartes et plans d'autres villes de France, d'Europe et d'autres continents, du passé ou de l'époque en question, correspondant à l'ouverture, dans les travaux de Poëte, vers une approche générale de l'urbanisme ${ }^{8}$. La pluralité des sources mobilisées par Poëte en tant qu'historien a déjà été analysée 9 . Cependant, la rencontre avec la collection iconographique semble confirmer l'idée que les images ont une place dominante dans le dispositif d'observation de l'auteur, autour de laquelle toutes les autres sources se déploient, et que ces images sont la source d'une singularité d'approche.

\section{Une vie de collection}

En soi, l'existence à cette époque d'une collection de clichés, ne saurait surprendre. Depuis presque 30 ans déjà, les cours d'histoire de l'art en Europe et en Amérique du nord avaient été illustrés dans les écoles des Beaux-Arts et les universités par des projections. Il s'agissait d'un moyen permettant de visionner ou de faire voir des œuvres, d'en présenter des détails comme "à la loupe ". Bien que visibles sous une forme "dégradée ", il est possible ainsi d'en faire une connaissance indirecte, à défaut de pouvoir être devant les œuvres elles-mêmes. On peut se poser la question de la perte - ou peut-être de la transformation? - subie par ces œuvres graphiques dessinées au trait noir, constitutives de l'essentiel de la collection. En effet, en étant photographiées, elles perdent en qualité de trait, et les qualités des fonds de

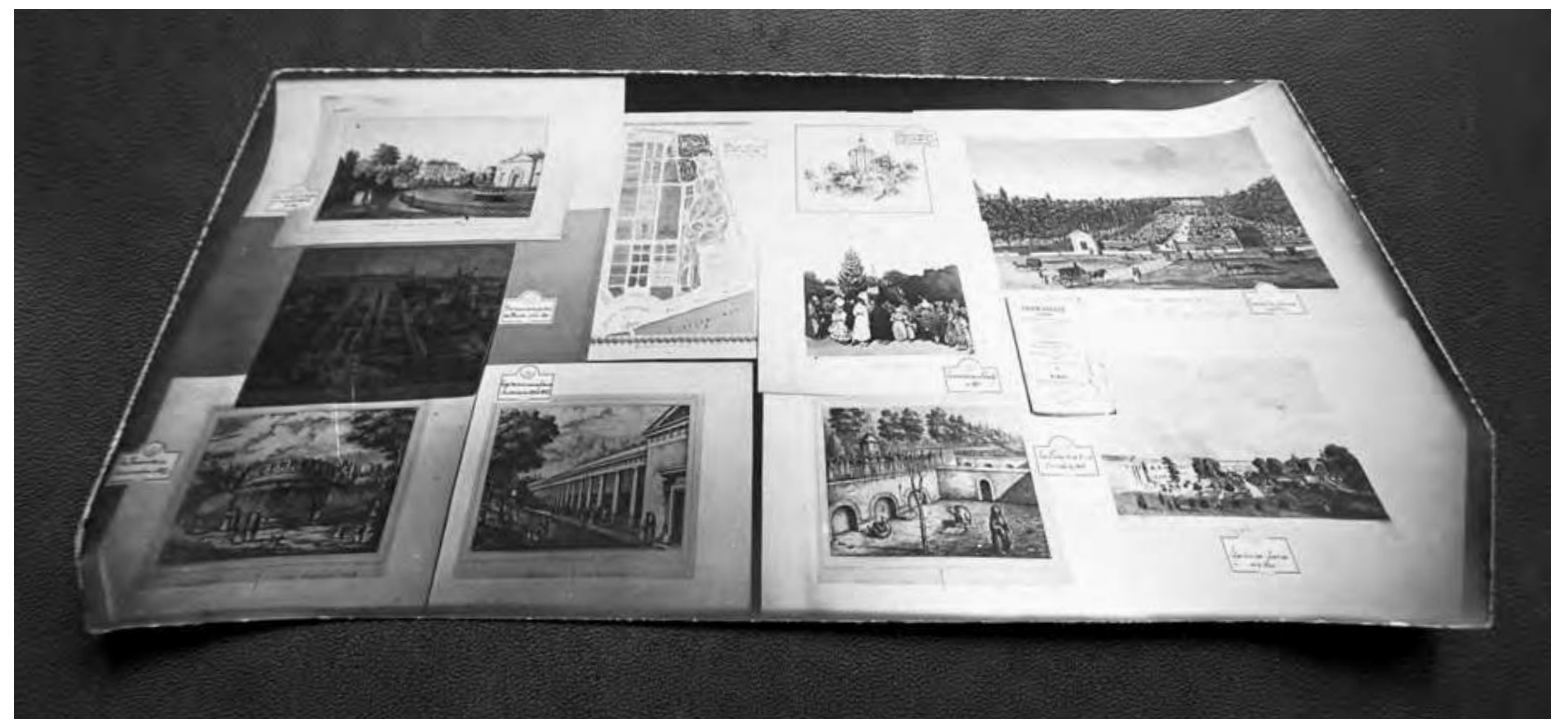

Vitrine horizontale, exposition de 1913, «Promenades et jardins de Paris " au sujet du Jardin des Plantes au XVII siècle. Photographie découpée en forme trapezoïdale suivant un effet de perspective. Pièce non classée, BHVP.

8. En 1917 et 1920, Poëte sera l'un des acteurs principaux, notamment auprès de I'homme politique Henri Sellier, dans la création de l'École des hautes études urbaines ainsi que de l'Institut d'histoire, de géographie et d'économie urbaines.

9. D. Calabi, op. cit. note 1 
papier s'effacent en faveur de ce qui, lors d'une projection, ne sera plus qu'un environnement de lumière blanche. Toutefois, bien plus que pour elles-mêmes, les images en question sont rassemblées et reproduites pour donner à voir la ville de Paris ainsi que sa population à travers I'histoire. On pourrait dire qu'il s'agit d'un outil de visualisation, voire d'un dispositif de visite : Poëte aurait tendance à dire de promenade, qui confronte la mémoire du spectateur des espaces à l'étrangeté de ces vues historiques de lieux, qu'il a l'habitude de traverser quotidiennement. De toute évidence, I'historien est sensible à la multiplicité de "spectacles vivants " du passé que la collection offre, et semble convaincu du fait que les expériences proposées peuvent affecter la perception des parisiens, quant à leur cadre de vie commun. Par ailleurs, on peut concevoir le trouble salutaire qu'un tel dispositif risque de présenter pour I'historien de Paris lorsqu'il éprouve la capacité propre de ses vues à narrer l'histoire de la ville. Même si pour lui ce ne serait que grâce à l'examen critique des documents, soit I'une des compétences fondatrices de ce que certains en ce début de siècle appellent la " science historique ", que ces vues rendaient véritablement leur richesses de "faits " précis et concrets. Il y a plus. En effet, bon nombre des œuvres en question proviennent d'albums ou de collections qui avaient précisément pour but de donner à voir la ville, que ce soit à un souverain, au touriste, à l'amateur d'histoire locale, à l'érudit ou aux acteurs d'une de ces transformations. Ainsi, l'archive de la BHVP est une sorte de collection de collections dans laquelle se rencontrent différentes associations de valeurs documentaires et esthétiques, permettant de suivre l'évolution des visibilités urbaines, voire d'envisager une archéologie du regard sur la ville. À travers sa carrière et à des degrés et des manières différents, chacun de ces registres sera exploré par l'instigateur de la collection iconographique de la BHVP.

Bien que connue grâce aux usages publics qui en avaient été faits, la collection n'a jamais été mise à la disposition du public. Comme Poëte I'indique discrètement en 1924, la collection aurait dû être disponible, dans les années ayant précédé la Grande Guerre, sous forme de catalogue imprimé $^{10}$. Or finalement, sa seule réelle présentation imprimée - partielle et techniquement peu aboutie - sera le volume intitulé "Album » d'Une vie de cité, publié un an plus tard ${ }^{11}$. Quoi qu'il en soit, au vu (notamment) de tous les aspects de son histoire évoqués plus haut, il convient de considérer une telle collection en tant qu'agent social possédant une capacité à configurer autour d'elle un réseau étendu de relations sociales et de produire, au sein de ce réseau, un certain nombre d'effets. Ce qui revient à considérer cette archive comme un

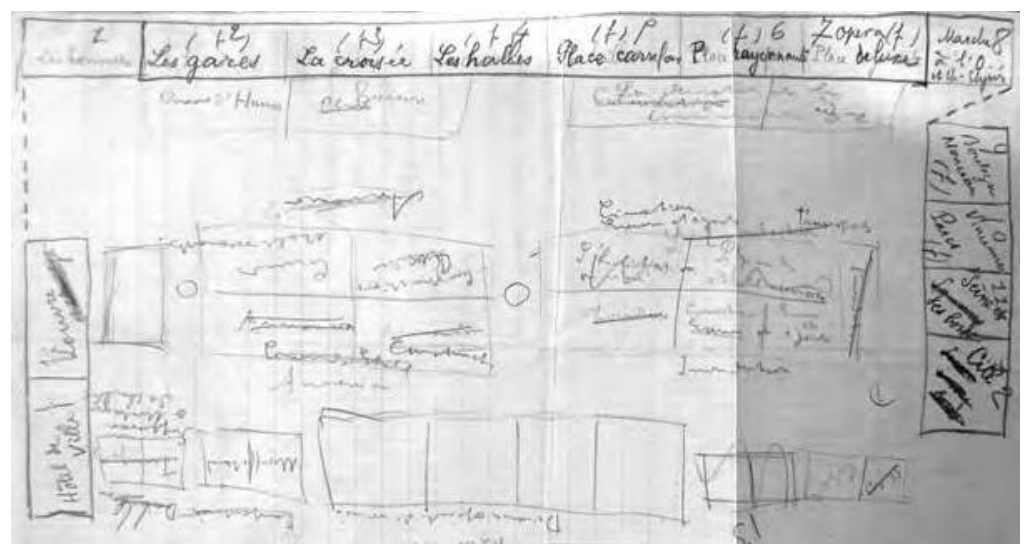

Exposition

de 1910, « La transformation de Paris sous le Second Empire ".

Plan annoté au crayon par M. Poëte (1909 ou 1910). Papiers Poëte, BHVP, MS 144, fol. 326

10. En effet, Poëte évoque « un catalogue de l'iconographie parisienne, dressé par cet établissement [la Bibliothèque historique de la Ville de Paris] dans les années ayant précédé la guerre de 1914 et qui allait être imprimé lorsque celle-ci survint », p. 9, Une vie de cité. Paris de sa naissance à nos jours, vol. 1, 1924.

11. Op. cit. note $3,1925$. 
protagoniste à part entière pouvant être le sujet d'un approche biographique, au même titre - et au prix des mêmes risques - que le personnage qui en est à l'origine ${ }^{12}$. Dans le présent contexte, il s'agit d'une part, de présenter le " protagoniste encore inconnu " de façon à en offrir une première approche et d'autre part, de reconsidérer le personnage avec qui il est étroitement associé. Face à la multiplicité propre à l'un comme à l'autre, on se limitera dans le présent contexte à la narration d'une série d'événements à travers lesquels leur relation s'est développée dans un premier temps: à savoir sept expositions annuelles d'histoire de Paris, toutes concernées à des degrés et de manière différents par les transformations urbanistiques à venir, qui ont eu lieu à la Bibliothèque historique entre 1907 et 1913. En effet, faute de pouvoir les recréer de toutes pièces, en croisant des annotations dans la Liste de projections et de rares documents photographiques, les renseignements offerts par les livrets de présentation préparés chaque année pour les visiteurs des expositions, par les notes de conférences et articles et, enfin, par les notes de préparation trouvées dans les papiers personnels de Poëte ${ }^{13}$, il est possible de se faire une bonne idée de la forme et des contenus de ces événements.

Suit une sélection de clichés de la collection présentant des documents ayant fait partie de ces expositions.
Cette sélection sera associée à des extraits des livrets et des articles de présentation des expositions, ainsi que des cours publics, intégrant des projections proposées pendant ces mêmes années par Poëte. L'approche des expositions sera faite par ordre chronologique, ce qui permet de révéler quelques aspects saillants de chacun des deux " protagonistes " sous l'influence de l'autre. Cela ouvrira quelques pistes pour mieux comprendre la profonde idiosyncrasie qui caractérise les modes d'action et les productions de Marcel Poëte: entre plusieurs disciplines, entre cultures savantes et culture populaire, entre des styles d'écriture scientifiques et littéraires, entre réflexion sur le passé urbain et la préparation de transformations futures de l'agglomération parisienne. En même temps, présenter et raconter les expositions revient à montrer quelque chose de la densité de relations d'ordre technique, matériel, social, culturel, politique qui se configurent autour de la création et des usages faits de la collection iconographique: on pourrait dire qu'elle en est l'indice ${ }^{14}$.

\section{Un format d'exposition particulier}

La photographie de l'exposition de 1907, installée dans une salle de I'hôtel Le Peletier de Saint-Fargeau ${ }^{15}$, une esquisse en plan de l'exposition de 1910 et une photographie de vitrine de l'exposition de 1913 (les trois étant reproduites ici), offrent un aperçu de ce qui était probablement le format général de l'ensemble des sept expositions, ainsi que d'autres initialement prévues par la Bibliothèque historique. Dans une série de vitrines à l'horizontale et derrière des portes vitrées d'armoires, sont présentés des regroupements thématiques d'images, estampes originales, livres illustrés ou reproductions photographiques d'œuvres graphiques, de tailles différentes, placées côte à côte, parfois posées l'une sur l'autre, agencées de façon à inviter les spectateurs à les parcourir de l'œil et à opérer des rapprochements. Des feuillets de papier portant des légendes sont parfois posés directement sur les agencements d'images pour présenter des titres ou des noms d'auteurs, ou bien pour désigner un rapprochement à faire entre plusieurs vues qui se côtoient. Quelques grands documents occupent les rares surfaces de murs qui ne sont pas recouverts par le mobilier. Si les déplacements du regard d'une image dans une vitrine vers les autres peuvent et doivent se démultiplier, partant librement dans des sens différents, le passage d'une vitrine à l'autre suit un ordre prédéterminé, correspondant aux chapitres des livrets qui accompagnent chaque exposition.

S. M.
12. À titre d'exemple, voir les différentes pratiques de l'object biography, dans Lorraine Daston, Things That Talk. Object Lessons in Art and Science, New York, Zone Books, 2004. Nous avons également étudié de nombreuses pratiques de la biographie d'objet chez les anthropologues de

la culture des quinze dernières anées.
13. Les Papiers Poëte : archive de 108 volumes, répertoriés dans le Catalogue des manuscrits de la BHVP. Don de Françoise Bardet, 1954. Ils se composent de notes de travail préparatoire à ses livres, articles, cours et conférences, de textes sur I'histoire urbaine et l'histoire de Paris.

14. Voir - par rapport à la notion d'indice Alfred Gell, L'Art et ses agents. Une théorie anthropologique [1998], tr. fr., Bruxelles, Les Presses du Réel, 2009.
15. II s'agit de l'hôtel particulier où se trouve le musée Carnavalet et où était logée, encore en 1907, la BTHVP. 



« La vie populaire à Paris par le livre et l'illustration ( $x v^{e}$ à $x x^{e}$ siècle) "

\section{Extrait du livret de l'exposition, 1907}

Exposition se déroulant dans un cadre d'anciens plans et aspects de Paris, et ouverte à l'Hôtel Le Pelletier de Saint-Fargeau, 29, rue de Sévigné, tous les jours, le dimanche compris, de 10 heures du matin à 5 heures du soir, depuis le 4 juin jusqu'au 1er octobre 1907.

Le vendredi, à 4 heures, présentation des pièces exposées, avec conférences sur la Rue parisienne, les Cris de Paris, la Chanson à Paris, les Fêtes parisiennes, les Sports et l'Aérostation à Paris, les Anciens plans de Paris, la Littérature populaire, I'Exercice des métiers.

Cette Exposition, installée dans la grande salle du Cours d'histoire de Paris, inaugure la série d'expositions annuelles prévues dans la nouvelle organisation du Service de la Bibliothèque et des Travaux historiques de la Ville de Paris. Elle est exclusivement formée de pièces appartenant aux diverses collections de ce Service, et est destinée à servir en quelque sorte de leçon de choses sur l'objet auquel elle se rapporte. Le Service a essayé, par des rapprochements entre le livre et l'image et par une notice explicative jointe à chaque pièce, de faire œuvre instructive dans le domaine de I'histoire parisienne. II s'est proposé, en outre, d'établir une sorte de chaîne, pour un même genre de pièces entre les époques anciennes et le temps présent. [...]
Vue du Pont-Neuf vers 1650. Gabriel Perelle (eau-forte).

Vue, " Le Pont-Neuf vue du côté de la rue Dauphine. L'embarras de Paris ». Nicolas Guerard (eau-forte) 1660.

" Autre vue particulière de Paris prise du Pont-Neuf... ". Eau-forte faisant partie d'un album de vues, milieu du XVIII' siècle.

\section{Vitrine 12 \\ Le Pont-Neuf}

Pendant tout le $\mathrm{XVII}^{\mathrm{e}}$ siècle et une partie du $\mathrm{XVIII}^{\mathrm{e}}$, on peut dire que le Pont-Neuf a incarné la vie populaire à Paris. Ses larges trottoirs, les premiers et de beaucoup, qui aient été ménagés sur la voie parisienne, attirèrent naturellement les oisifs et les promeneurs trop à l'étroit dans les autres rues où rien ne leur offrait un refuge contre les carrosses. Les petits commerçants, les étalagistes suivirent le courant et vinrent exposer leur marchandise autour du «Cheval de bronze » d'Henri IV ou le long des bâtiments de la pompe de la Samaritaine. Une estampe coloriée de Perelle, une photographie d'un tableau de Ragunet permettent de se faire une idée de ces boutiques en plein vent qui on survécu en cet endroit au moins jusqu'au second empire. [...] 


\section{"Vie populaire ", " vie urbaine "}

Dès 1907, les expositions s'adressent à un public hétérogène comprenant de simples curieux, des historiens et collectionneurs amateurs, mais aussi des universitaires curieux de connaître un corpus de sources et une nouvelle méthode de travail ${ }^{1}$. S'appuyant sur des sélections représentatives de vues, l'exposition précise trois notions qui fonderont durablement l'approche de I'historien. Il s'agit d'abord, classiquement, de la topographie historique : soit des mises en relation de cartes anciennes de Paris et de ses quartiers, de vues et parfois de textes permettant de suivre le développement historique du " cadre " physique parisien. L'association étroite de ces cartes à des photographies ${ }^{2}$ datant des dernières décennies du $\mathrm{XIX}$ siècle et du début du $\mathrm{XX}$ permettent de mesurer l'importance des transformations ayant eu lieu depuis 1850. II s'agit ensuite de ce que Poëte désigne ici comme la vie populaire et qui, sous une forme élargie, deviendra plus tard la vie urbaine $^{3}$ : autrement dit des configurations de relations dynamiques et changeantes qui animent les rues et les lieux publics en associant des personnes entre elles mais aussi avec des objets et parfois des animaux. Elle est figurée depuis le $\mathrm{xv}^{\mathrm{e}}$ siècle à travers une diversité de genres d'images : des paysages (ou « vues ") remplis de personnages voire de foules, de scènes d'individus connus et de personnages "typiques". Les pièces présentées offrent une démonstration concise de la capacité de restitution de l'épaisseur de cette "vie ", qu'une accumulation conséquente d'images permettrait de réaliser, époque par époque. Cette restitution se déploie non seulement dans l'espace mais aussi à travers des temporalités - quotidiennes, saisonnières - qui rythment les existences, et englobe les situations de travail, de loisirs ou de fêtes, ainsi que les expériences liées aux déplacements (" les moyens de transport "). Sont présentés quelques exemples d'objets, d'activités ou d'événements (les cabarets et cafés, les sports, les montgolfières etc). Sont également présentés des moyens par lesquels circulent les informations et les images (l'affiche, la pièce volante, le canard, la chansons etc.). En effet, ce point fait écho aux demandes réitérées par Marcel Poëte auprès des " amis " de la bibliothèque de recueillir des pièces d'actualité afin de les déposer à la BHVP. Il est difficile de ne pas faire le lien entre cette initiative de collecte et l'enthousiasme, largement répandu, pour les images imprimées, notamment les photographies coloriées diffusées surtout grâce à des magazines illustrés, d'invention récente. Il convient aussi d'évoquer ici la mode toute récente à l'époque de l'usage domestique de la lanterne magique à ampoule électrique, invention qui avait rendu plus simple et plus sûr l'usage de tels instruments, et que I'historien utilisera lors de ses conférences ${ }^{4}$. II convient par ailleurs de rappeler le succès, toujours vif en 1907, du diorama ainsi que le cinéma, encore à ses débuts. Et enfin, il faut citer la carte postale illustrée qui, dès son introduction en France en 1906 connaît un succès fulgurant ${ }^{5}$. Pour l'historien, topographie et vie urbaines se renvoient l'une à l'autre, se définissent mutuellement. Il désigne la globalité qu'elles constituent par le terme physionomie. L'exposition insistera sur le Pont-Neuf en tant que lieu central de vie populaire, d'une certaine manière fondatrice pour la " grande ville » de Paris dès la fin du $\mathrm{XV}^{\mathrm{e}}$ siècle. Le lieu gardera ce caractère pendant presque 200 ans (comme en témoignent de nombreuses vues dont celles présentées plus haut) jusqu'à ce que, lors des rénovations haussmanniennes, cette partie centrale de la physionomie de la ville " meurt " au profit de la « naissance " d'autres, situation que l'historien rapporte sans la moindre nostalgie ou regret. Du reste, de tels changements feraient partie, selon ses termes, d'un lent processus marqué de résistances, de changements lents et de transformations puissantes, désigné dès 1907, dans des ouvrages qu'il publie indépendamment de l'exposition, par le terme d'évolution urbaine. Le sens de l'évolution urbaine, écrit Poëte cette même année dans son livre, Enfance de Paris ${ }^{6}$, est nécessaire en vue de " préparer l'avenir ».

S. M.
1. Les résumés de certaines conférences conservés dans la collection des Papiers Poëte à la BHVP, s'accompagnent de listes $\mathrm{d}^{\prime}$ 'historiens et de chartistes qui étaient présents. On y trouve les noms de certains historiens connus dont Georges Espinas et Georges Huisman. Le nombre total d'auditeurs aurait été, en moyenne, de l'ordre d'une centaine de personnes.

2. Parmi celles-ci figurent des photographies sélectionnées de l'œurre complète de
Charles Marville qui avaient été acquises par la Ville de Paris.

3. Ce terme sera notamment utilisé pour nommer la revue de l'Institut d'histoire, de géographie et d'économie urbaine dont le premier numéro date de 1920.

4. La société Molteni, à qui la BHVP commande la photographie et la fabrication des projections, fait à l'époque le gros de sa fortune considérable grâce à la vente de clichés aux sujets divers ainsi que de lanternes magiques destinés à l'usage au foyer.

5. Comme le raconte un article de 1907 de la Revue politique et littéraire, I'apparition de cette invention avait déclenché une crise de fonctionnement sans précédent du système postal français pendant l'été 1906.

6. Voir page 2, Enfance de Paris. Formation et croissance de la ville, des origines jusqu'au temps de Philippe Auguste, Paris, Colin, 1908. 

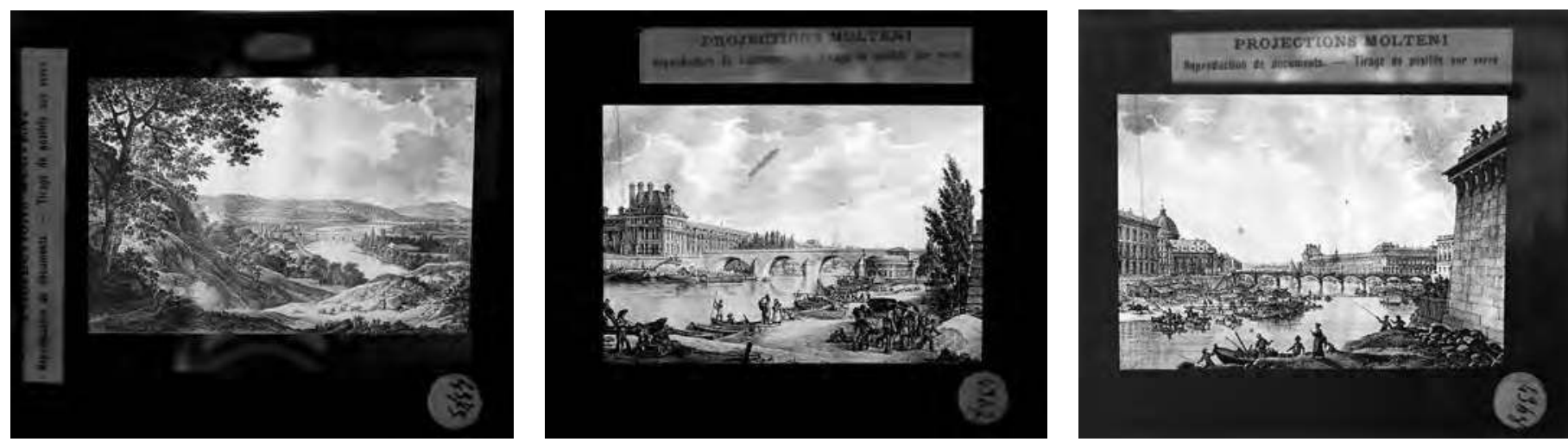

\section{«Au Temps des Romantiques. L'image de Paris », (1907) \\ Extrait}

C'est proche et pourtant déjà lointain, comme un paysage qu'à distance on regarde, après l'avoir traversé : les détails se noient indistincts dans l'ensemble qui varie suivant le point de vue ou au gré de la lumière capricieuse. Essayez de saisir cette image de Paris à travers les écrits de l'époque, les gravures ou dessins, les souvenirs des contemporains subsistants. Vous ne parviendrez qu'à tracer les lignes fugitives de formes qui changent. Artistes ou auteurs nous montrent la ville telle qu'en eux ils l'ont vue: c'est dans la diversité et la mobilité de leurs impressions qu'elle se dessine.

À l'aurore du Romantisme, les lithographies de Marlet, de 1821, la série de Chalon, de 1822, expressive dans sa grâce naïve, représentent plutôt les mœurs que les aspects topographiques. Ce sont au contraire des vues que retracent, en 1818, le recueil du peintre-graveur DamameDémartrais, élève de David, et, en 1822, celui du général Louis Bacler-d'Albe. II y a plaisir à se laisser conduire par ces deux guides dans le Paris de la Restauration.
En des planches qui sont, pour ainsi dire, des aquarelles gravées, Damame-Démartrais nous permet de suivre notamment la Seine, depuis le pont d'Austerlitz jusqu'à Passy. La voici qui miroite au soleil, offrant aux voyageurs du coche d'Auxerre, que tirent péniblement quatre robustes chevaux, le spectacle du port et du quai Saint-Bernard, avec la masse des frondaisons du Jardin du Roi, et là-haut, en plein ciel, le dôme de l'église Sainte-Geneviève ; au nord, l'île Louvier défile, dressant ses chantiers de bois à brûler qui servent à l'approvisionnement des habitants du Marais. "Les bords en sont très agréables et très pittoresques. Ils invitent les curieux et les solitaires à s'y promener ». Un petit pont de bois - le pont Grammont - qu'on a soin de fermer à la chute du jour, unit au quai, où s'élève l'Arsenal cet îlot, qui, d'autre part, se prolonge, à ses extrémités, par deux "garres» ou estacades "en énorme charpente ». Ces jetées, qui livrent passage aux barques sont destinées «à parer aux nombreux accidents des glaces ». Celle de l'est aboutit en face des Greniers de réserves ou d'abondance, dont 

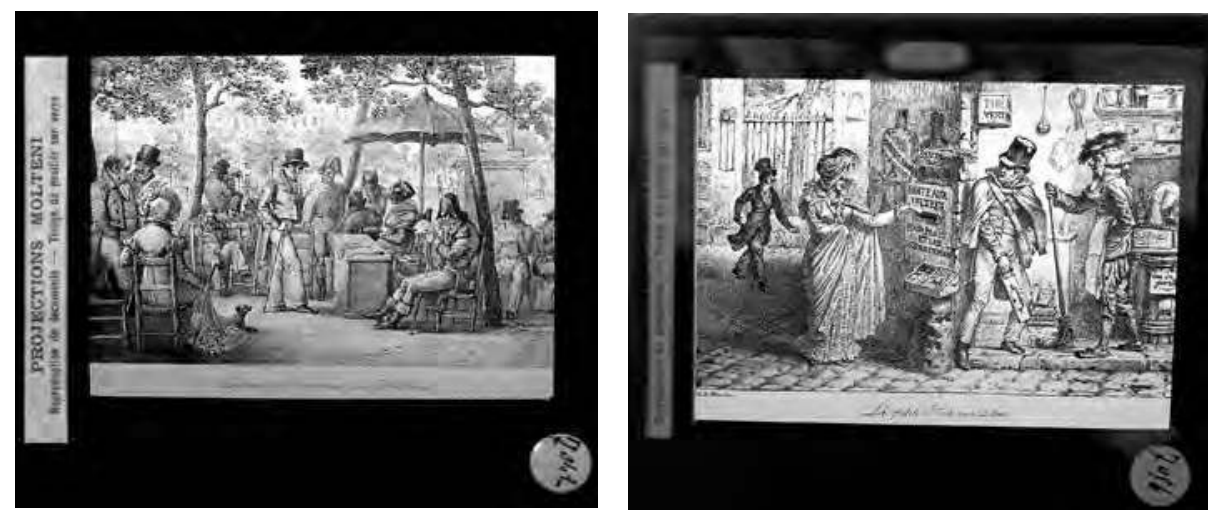

les cinq pavillons se profilent au long du nouveau boulevard Bourdon et de ces anciens fossés de la Bastille qui ne tarderont pas à recevoir le canal de I'Ourcq. L'estacade occidentale rejoint l'île Saint-Louis et forme, en quelque sorte, l'entrée du port Saint-Paul, d'où partent les coches de Corbeil, Nogent, Briare. L'archipel se termine par l'île de la Cité, que lie à la précédente le pont de la Cité, réservé aux piétons, qui payent cing centimes de péage. L'ancien cloître Notre-Dame regarde le Port-au-Blé et la Grève où, devant l'Hôtel de Ville que borde au sud la rue de la Mortellerie, ont lieu les réjouissances nationales et les exécutions. Au midi, la cathédrale, à laquelle s'attache le palais de l'Archevêché, domine le Port-aux-Tuiles, dont l'horizon est borné, à l'ouest, par le pont couvert de l'Hôtel-Dieu. De ce côté, l'île finit, avec la terrasse de l'archevêque, en une tache verdoyante. À son autre extrémité, c'est, au pied de la nouvelle statue de Henry IV, une langue de terre déserte, toute frêle sous la caresse de l'eau...

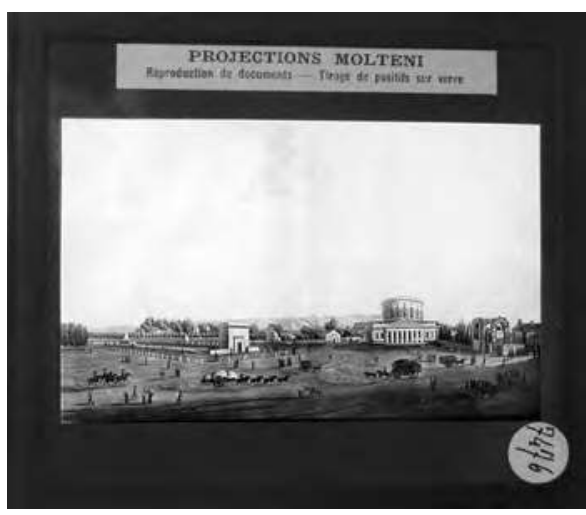

" Javelle ». Première de trois lithographies faisant partie de I'album Promenades pittoresques et lithographiques dans Paris et ses environs.

Louis Bacler-d'Albe, 1822.

"L'allée du bord de l'eau " [vue vers le Pont-Royal et le Louvre].

"Le Pont des arts" [vue depuis le Pont-Neuf].

« Lecture des journaux aux Tuilleries ". Première de deux lithographies faisant partie de I'album Tableaux de Paris. Jean Henry Marlet, 1821.

" La petite poste une lettre ".

"Vue de la barrière Saint-Martin ". Aquatinte faisant partie de l'album Paris et ses alentours, à plus de trente lieues à la ronde. Michel François Damame-Démartrais, 1818. 
Cette première d'une série de trois expositions constituant une séquence chronologique présentera non pas une époque de l'histoire de la ville ou de I'histoire politique du pays, mais une période allant grosso modo de 1800 à 1840 correspondant à une ère de la production et de la diffusion de genres artistiques. $\mathrm{Ce}$ tournant du siècle qui correspond à l'essor du romantisme dans l'art, sera ainsi présent dans l'exposition à travers des estampes tirées d'albums commandés le plus souvent par des galeristes, à l'attention d'un public de collectionneurs. Selon Poëte, il s'agit d'une phase qui se termine avec la mort du romantisme, attribuable à la fascination générale pour le daguerréotype. Aux gravures vont se rajouter, à partir de 1818, des lithographies destinées également à un marché d'amateurs. Ces images sont souvent coloriées, délicatement mais librement, à l'aquarelle. Il s'agit, semblerait-il, d'œuvres ne pouvant pas toujours être considérées comme pertinentes pour les collections publiques, car étant d'origine récente et destinées avant tout au commerce et au divertissement ${ }^{1}$. En effet, pour monter I'exposition, il aura fallu s'appuyer sur l'enthousiasme et sur les moyens financiers de collectionneurs amateurs, membres d'une toute nouvelle Société d'iconographie parisienne fondée en 1907. Ce sont eux qui ont fourni une majorité des pièces originales qui seront présentées, aux côtés de quelques imprimés de la BHVP2 Les œuvres romantiques dont il est question tendent plutôt vers une figuration " vraisemblante " (selon le terme repris par Stephen Bann ${ }^{3}$ ), visant à susciter des effets de réel. Elle se caractérise par une narrativité descriptive constituée d'effets fuyants, d'ensembles pittoresques et de captations de détails ou d'incidents anecdotiques destinés à apporter des touches de " couleur locale ". Quant aux sujets abordés, ils sont souvent historiques : l'art pictural comme le roman, s'emploient à "faire revivre " des scènes d'histoire. Ce n'est pas le cas dans la présente exposition : cependant, les « lieux de la vie romantiques » qui s'y trouvent figurés sont souvent caractérisés par des irrégularités pittoresques évocatrices des couches d'histoire. Quoi qu'il en soit, c'est la promenade à travers Paris promise par le titre de l'exposition qui est - ceci à double titre le fil conducteur à travers lequel le spectacle « vivant " présenté par les œuvres se déroule. Il s'agit d'abord d'une pratique sociale de l'époque, que donnent à voir de nombreuses vues présentées le plus souvent sur les boulevards (qui, à cette époque, commencent à se transformer en véritable " espace urbain ") ou sur les quais de la Seine. Il est également question de " se promener ", avec les yeux, le long des chemins ou des routes (de terre ou d'eau); de " traverser " avec imagination une image pour arriver au point depuis lequel une autre vue sera proposée (voir par exemple la séquence des trois vues de Bacler-d'Albe). Ainsi, se déroule, à travers la promenade imaginée, une séquence d'emplacements dans le cadre urbain d'où on perçoit des scènes pittoresques de vie, renforcées par des évocations tirées de textes d'époque évoquant ce que les vues donnent à voir. Le long de ces promenades de plaisirs imaginaires, des renseignements documentaires sur les complexités de la physionomie urbaine se dévoilent. C'est sans doute la première manifestation chez I'historien de ce qui sera désormais une approche récurrente visant une description dense et résolument empirique des espaces urbains. Par la suite, non seulement ses expositions mais aussi nombre de ses conférences, de ses textes d'histoire, de ses guides et même de ses scénarios de films regorgeront de descriptions recueillies comme en avançant in situ, souvent avec des indications précises de trajectoires à suivre, voire des indications sur l'orientation du regard à adopter par rapport à des points particuliers. Par ailleurs, Poëte ne se prive pas de I'usage de moyens "littéraires". En effet, les observations le long des
1. C'est le cas notamment d'un artiste comme Shotter Boys, dont les aquarelles plairont à Poëte grâce au naturel avec lequel il saisit des pratiques ordinaires de la rue. Par contre, les vues de

Damame-Démartrais ou de Charlet qui sont dans l'exposition figuraient déjà à l'époque dans des collections publiques.

2. La société se réunit régulièrement à I'Union des arts décoratifs pour discuter des pièces de diverses époques acquises par les uns et les autres, et pour préparer annuellement la publication d'un recueil de reproductions de documents graphiques (à l'origine des estampes, des lithographies, ou des dessins). Présent fréquemment à ces réunions (comme l'indique le Bulletin de la Société d'iconographie parisienne dès 1907), Poëte profitera de leurs collections pour découvrir des pièces nouvelles et en échange, il mettra à disposition de la société ses connaissances spécialisées.
3. Voir Stephen Bann, The Clothing of Clio. A Study of the Representation of History in 19th Century England and France, New York, Cambridge University Press, 1984.

4. Augustin Thierry, Lettres sur I'histoire de France, Paris, Sautelet, 1827.

5. Voir Pierre Rosanvallon, Le Peuple introuvable. Histoire de la représentation démocratique en France, Paris, Gallimard, 1998 ; Marcel Gauchet, « Les Lettres 
promenades virtuelles à travers les images seront livrées parfois sous forme de fictions - toutefois très précisément construites sur le plan de la véracité historique - autour d'un protagoniste historique. En effet, il serait difficile de ne pas voir de similarité entre le mode d'écriture " littéraire " que Poëte adopte dans ses premiers ouvrages et articles datant de 1907 et 1909, et certaines manières d'écrire l'histoire des années 1820, d'autant plus qu'il s'agit, dans beaucoup de cas, des tous premiers textes d'un genre nouveau d'histoire locale. Comme l'a montré Stephen Bann à travers ses écrits sur les genres de peinture historique de l'époque, des relations complexes lient l'art visuel avec l'historiographie dans la première moitié du XIX ${ }^{\mathrm{e}}$ siècle. En ce qui concerne Poëte, son affection pour Augustin Thierry, historien des villes et du tiers état ${ }^{4}$, est bien connue et souvent rappelée - paraît-il - à son entourage. À y regarder de plus près, on remarque des similarités d'intention et de style d'écriture entre I'historienconservateur en chef de la BHVP et I'historien de la monarchie de Juillet, prônant une historiographie susceptible de participer à la construction de la connaissance d'une population par elle-même ${ }^{5}$. Chez Poëte, comme c'est le cas sous une forme différente chez Augustin Thierry dans les années qui précèdent 1830, l'écriture de l'histoire locale se positionne entre la narration destinée à faire sentir un lien avec le passé d'une population urbaine dont on fait partie, et une volonté de prise de distance réflexive.

En effet, malgré le sérieux reconnu de ses travaux d'archiviste et la profondeur de sa connaissance de I'histoire de Paris, la tentation littéraire à laquelle Poëte cèdera souvent - en témoigne son refus de citer des sources sous forme de notes en bas de page - lui porte préjudice auprès d'une communauté scientifique soucieuse d'assurer, et ceci au prix de quelques difficultés réelles, la qualité désintéressée de ses recherches. Cette communauté est également soucieuse de la nature des rapports qu'elle entretient avec ses aînés du $\mathrm{XIX}^{\mathrm{e}}$ siècle : or si Thierry peut être considéré comme un père fondateur de la " science historique ", il ne saurait plus être question pour un historien sérieux de suivre son exemple $^{6}$. Les raisons de la transgression de Poëte sont difficiles à cerner de près. Cependant, il est certain qu'elles sont liées à sa volonté, en tant que conservateur en chef de la BHVP, $d$ 'agiter, d'une manière qui fait penser à la notion bergsonienne $d^{\prime}$ '" imagesouvenir ", une sorte de mémoire vive parisienne au moyen du texte imagé et de l'image, jusqu'à ce qu'elle dévoile des possibilités de vie nouvelle.

S. M. sur l'histoire de France d'Augustin

Thierry " dans Pierre Nora (dir.), Les

Lieux de mémoire, t. II, "La Nation », vol. 1, p. 217-316, Paris, Gallimard, 1986,

Bibliothèque illustrée des histoires ; Rulon

Nephi. Smithson, Augustin Thierry: Social and Political Consciousness in the Evolution of a Historical Method, Genève, Droz, 1973.
6. Voir les commentaires de Camille Julian au sujet d'Augustin Thierry dans son ouvrage de référence : Extraits des historiens $d u$ XIXe siècle, publiés, annotés et précédés d'une introduction sur I'histoire de France, Paris, Hachette [1897]. 

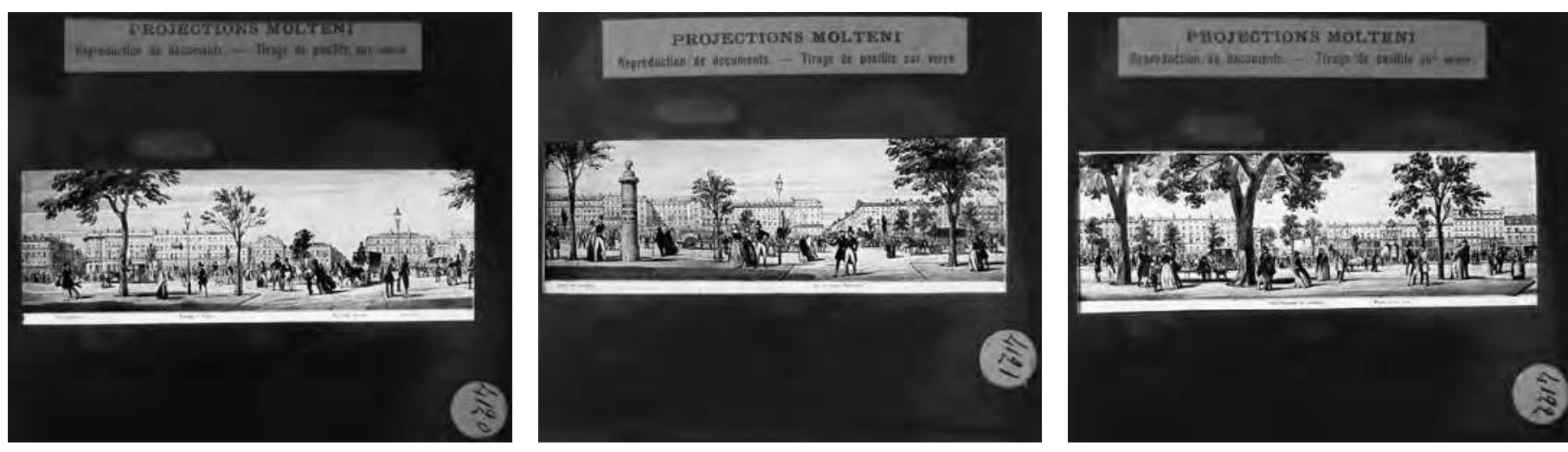

\section{Livret de l'exposition « La transformation de Paris sous le Second Empire » (1910) Extrait}

Qu'est-ce que l'art de la ville? C'est l'ensemble des règles applicables aux conditions d'existence et de développement d'un centre urbain. Ces règles, si elles trouvent leur application immédiate dans le cas d'une de ces villes modernes bâties de toutes pièces et dont les États-Unis nous offrent maints exemples, ne sont, pour les vielles cités, que le résultat d'une lente évolution des siècles. Elles commencent à se dégager au $x V^{e}$ siècle, en ce qui touche Paris. Et c'est sous l'influence du classicisme de la Renaissance qu'apparaissent, avec le « droict allignement » des nouvelles voies, la régularité, la symétrie des constructions, « l'ordre [...] rare et beau de ces grands bâtiments » qui font la parure du Paris de Louis XIV. Sous le règne de ce dernier naît cette grâce urbaine: la ligne sinueuse de la promenade des boulevards à laquelle se rattache une grande part des "embellissements » du xvIII siècle [...] Loin de mettre un terme à ces projets, le mouvement révolutionnaire leur donna corps [...]

Du réseau des voies créées il est permis de dégager des unités topologiques comme la gare, la Croisée, la Halle auxquelles s'ajoutent ces points de rattachements de voies: la place carrefour, tels la Bastille et le Château d'Eau (République), confluents de courants de circulations intenses; la place rayonnante, comme l'Étoile et le Trône (Nation), artificielles créations d'embellissements; le carrefour de luxe qui, en matière urbaine, caractérise l'époque en faisant du théâtre de luxe par excellence: I'Opéra, un centre topologique. Et ce luxe va à l'ouest où l'entraîne encore la naissance de toute une ville au long des somptueux boulevards et avenues qui transforment les quartiers de Monceau, de l'Étoile, de Chaillot. En ce dernier lieu, le baron Haussmann qui s'est peut-on dire, fait la main dès le début de son administration, par le nivellement des abords de la Tour St-Jacques, n'hésite pas à supprimer le vieux «mont de Chaillot». Sa main dévastatrice autant que créatrice transforme, dans un autre endroit, la fourmilière humaine de la cité en une solitude que peuplent de mélancoliques bâtisses administratives [...]

Le Paris nivelé, régularisé, sous le passage dominant de "voies romaines», c'est à tous égards un Paris transformé. On n'a peut-être pas suffisamment déduit toutes les conséquences sociales d'une pareille œuvre édilitaire. Ainsi transplantée, la mentalité des habitants ne doit-elle pas finir par subir des modifications? Ajoutez à cela le développement des chemins de fer et vous entreverrez tout ce qu' « un plus grand Paris » devait entraîner. La ville, moins chérie des Parisiens qui se sentent plus distants les uns des autres et la quittent durant une partie de l'année, devient un lieu cosmopolite qui a sa saison. Et si vous considérez qu'il s'agit de la capitale du pays le plus centralisé qui soit, l'horizon des conséquences s'élargira devant vous.

Relevons, en outre, le déplacement de population que les boulevards d'Haussmann ont amené dans 



la ville: si les gens riches s'en vont alors peupler les somptueux quartiers de l'ouest, la classe pauvre, les ouvriers, qui se voient chassés du centre de Paris par la maison bourgeoise qu'on y élève, se dirigent au nord, à l'est et au sud de la ville.

Comme tout tient à tout, c'est le temps où naissent les hôtels caravansérails, les grands magasins, les "cafés aux vingt-quatre billards». De cette transformation générale, la présente Exposition essaye de donner une idée, avec l'aide d'artistes consciencieux qui nous ont laissé des vues diverses du Paris de ce temps, avec aussi la précieuse ressource de photographies de cette époque (Marville, Richebourg, etc.). Plusieurs photographies ont été rapprochées, pour la même vue, de gravures et montrent la fidélité documentaire des eaux-fortes de Martial par exemple, ou de bois d'illustrations. L'abondance de ces derniers se justifie par le rôle de renseignements qu'ils jouaient auprès du public en un temps où la photographie n'était pas encore entrée dans les procédés du journal. Des dessins originaux, œuvres de Thorigny, Fichot, Trimolet, etc., voisinent avec ces pièces variées.

Telle qu'elle se présente à ses bienveillants visiteurs, cette Exposition ne s'adresse pas moins à ceux qui sont soucieux de l'avenir qu'aux curieux du passé. II n'est certes pas possible de bien connaître l'historie topographique de Paris au cours des siècles écoulés, si l'on ne s'est point familiarisé avec les bouleversements d'Haussmann. Mais il n'est pas davantage possible d'envisager, en toute connaissance de cause, les conditions actuelles et futures d'existence de la ville, sans avoir présent à l'esprit, le grand mouvement de transformation, sujet de cette Exposition. Il est dès lors permis de s'étonner de l'insuffisance absolue des moyens d'informations historiques dont on dispose sur le Paris contemporain. Si bon nombre de documents ont péri dans l'incendie de I'Hôtel de Ville en 1871, combien n'en reste-il pas en divers lieux ou dépôts, qui devraient faire l'objet de dépouillements systématiques, puis de répertoires à I'usage des administrateurs autant que des historiens! [...] De semblables œuvres permettraient d'assurer au travail municipal la base historique qui lui fait présentement défaut. Puisse cette modeste Exposition y aider!

\section{M.P.}

Trois sections d'un panorama datant de 1860.

"Vue de la Seine prise du Louvre". Lithographie faite d'après une photographie faisant partie de I'album (divers artistes) Paris dans sa splendeur, 1860.

Photographie de rue lors des démolitions de la période des rénovations haussmanniennes. Photographe des Travaux historiques de la Ville.

Immeuble, rue Tixandier. Lithographie, Adolphe Martial (autour de 1867). 


\section{Visualiser la transformation}

Au cours de l'année 1910, un changement net de perspective affecte I'ensemble de l'activité de la Bibliothèque historique.

Par décret du préfet ${ }^{1}$ et $d^{\prime}$ 'après une idée de Poëte, est créé un "Office de la vie municipale " dont la mission principale est le recueil et la publication, sous forme de catalogue raisonné annuel, des décisions de l'Hôtel de Ville², rajoutant ainsi à la mémoire des temps présents voulue par Poëte une dimension supplémentaire. Alors que la séquence chronologique des expositions qui était arrivée précédemment aux événements de $1848^{3}$, dans l'exposition intitulée « La Transformation de Paris sous le Second Empire ", l'évocation imagée de la vie urbaine cède quelque peu sa place centrale à un effort de construction de la notion de "l'art de la ville".

Dans cette année 1910 qui précède celle de la création de la "Société française des architectes urbanistes ${ }^{4}$, ce que I'on semble viser c'est l'identification de quelques fondements des futures pratiques de cette profession, notamment. Chez Poëte, il semblerait être avant tout question d'apporter une épaisseur proprement historique aux discussions de ce milieu (notamment dans le secteur du Musée social ${ }^{5}$ ) qui, selon lui, avait jusque là fait défaut. Une longue introduction dans le livret de 1910 présentant la première partie de l'exposition retracera les origines de I'" art de la ville " à la fin du $x v l^{e}$ siècle, basée sur l'application de ligne droite et le dégagement de grands " espaces libres " à travers lesquels on circule mais où, également, on " prend de I'air ". Ce moment de rationalisation de la forme urbaine serait, selon I'historien, le moment de l'apparition du " sens de la beauté " urbaine qui, du reste, serait incarnée dans les vues et les plans gravés de l'époque. L'exposition de 1910 évoque ensuite la " naissance sur le papier ", dans le Plan des artistes, du « Paris nouveau " puis déroule une histoire topographiquement précise des rénovations haussmanniennes, inscrite dans la perspective générale de la vie urbaine en état de transformation. La perspective physionomique des expositions précédentes est reformulée ici sous forme d'une approche qui présente, un par un, des ensembles complexes de constructions et de configurations de vie urbaine appelés " unités topologiques ". Ces unités incarnent des dimensions structurales à l'échelle de l'ensemble du Paris nouveau ("les gares", "les carrefours ", " les foyers de luxe "). En effet, c'est à travers la création, la modification ou la destruction de ces " unités topologiques " que l'essentiel du tableau de la transformation urbaine se dessine. Il est complété par l'évocation de certaines nouvelles infrastructures ainsi que des systèmes de services. Aux transformations intérieures de Paris, se rajoutent les extensions vers l'ouest et l'annexion des communes avoisinantes. L'exposition s'achève sur une évocation du problème du logement subi par les classes populaires, ainsi que les transformations qualitatives liées au saut d'échelle et à la modernisation qui ont touché des établissements publics et privés aussi bien que les rues de la ville. Ce qui permet de poser la question des conséquences sur les " mentalités" du changement convulsif dans une période de profonde transformation urbaine. L'apparition fortuite du terme surprend dans la mesure où il apparaît dix ans avant que la notion de mentalité ne soit approfondie par I'anthropologue Lévy-Bruhl pour être ensuite appliqué dans le champ de I'histoire, notamment par Lucien Febvre et Marc Bloch. Le rapprochement n'est pas gratuit en ce sens que, dans le livret, le terme est utilisé pour désigner des dispositions psychologiques pesant sur une situation historique, soit sensiblement la définition durable qui lui sera attribuée plus tard 6 . Par ailleurs, certaines questions posées dans une note synthétique d'exposition
1. Il s'agit de Justin de Selves qui occupera le poste de préfet de 1896 à 1911.

2. Voir le Bulletin de la Bibliothèque historique de la Ville de Paris de 1907.

3. L'exposition de 1848, est, pour des raisons d'espace, omise de la présente présentation

4. Voir Jean-Pierre Gaudin, L'Avenir en plan, technique et politique dans la prévision urbaine 1900-1930, Seyssel, Champ Vallon, 1985.

5. Voir Janet Horne, Le Musée social. Aux origines de l'État providence, préface de Pierre Rosanvallon, Paris, Belin, 2004.

6. En effet la notion de mentalité recevra sa première formulation approfondie en 1922 dans un livre célèbre de Henri Lucien Lévy-Bruhl, La Mentalité primitive, qui sera suivi deux ans plus tard par l'apparition de la notion $\mathrm{d}^{\prime}$ " histoire des mentalités " associée chez Marc Bloch et Lucien Febvre.

7. Comme des remarques écrites dans les Papiers Poëte le démontrent, I'historien prend connaissance de l'ouvrage peu de temps après sa publication, et y attribue une importance réelle. Dès 1920, Maurice Halbwachs appartiendra à I'Institut d'histoire, de géographie et d'économie urbaines. Dans son article de 1921 dans La Vie urbaine le sociologue revient sur 
rappelle aussi des idées de l'économiste et sociologue Maurice Halbwachs, concernant les déplacements de populations lors de la période des rénovations qui avaient été présentées deux ans plus tôt dans Les Expropriations et le prix des terrains à Paris. À partir de 1913, Marcel Poëte entretiendra, du moins par textes interposés, un véritable dialogue avec l'économiste et sociologue ${ }^{7}$. C'est donc dans la séquence de 1910 qui se termine en 1913 que Poëte se trouve comme rarement " dans le vrai " de sa profession d'historien et, plus généralement des sciences sociales et humaines de l'époque. On dispose de peu de renseignements précis concernant le choix de pièces exposées en 1910. Mais on trouve plusieurs types de vues conservées dans la collection iconographique datant du Second Empire. Les pièces en question témoignent d'une transformation du champ de la représentation urbaine. Désormais, il semble qu'une série d'écarts, voire d'oppositions s'expriment à travers une diversité de contenus et de modes d'expression.

Des caricatures porteuses de critiques sociales et politiques des rénovations en cours (dont un grand nombre de Daumier) contrastent avec des vues spectaculaires tirées des albums présentant des ensembles monumentaux de la ville (dont Paris dans sa splendeur, album de 1861); ou encore des vues photographiques de Marville ou sous forme de lithographies de Marlet, entre lesquelles Poëte invitera à la comparaison en termes de valeur documentaire; ou des vues de constructions et de rues du Paris nouveau versus des œuvres graphiques ou photographiques conservant des traces du vieux Paris, qui disparaît davantage jour après jour. Enfin, les statistiques graphiques font leur apparition au cours de cette exposition dans le livret de présentation $^{8}$ et avec elles, des modalités de raisonnement abstrait atypiques pour I'historien-promeneur, prônant des modélisations statistiques des aspects économiques et sociaux.

S. M.

I'aperçu historique de Poëte pour le mettre en relation avec les hypothèses de son ouvrage de 1908 .

8. II s'agit de statistiques d'usage des lignes d'omnibus. 

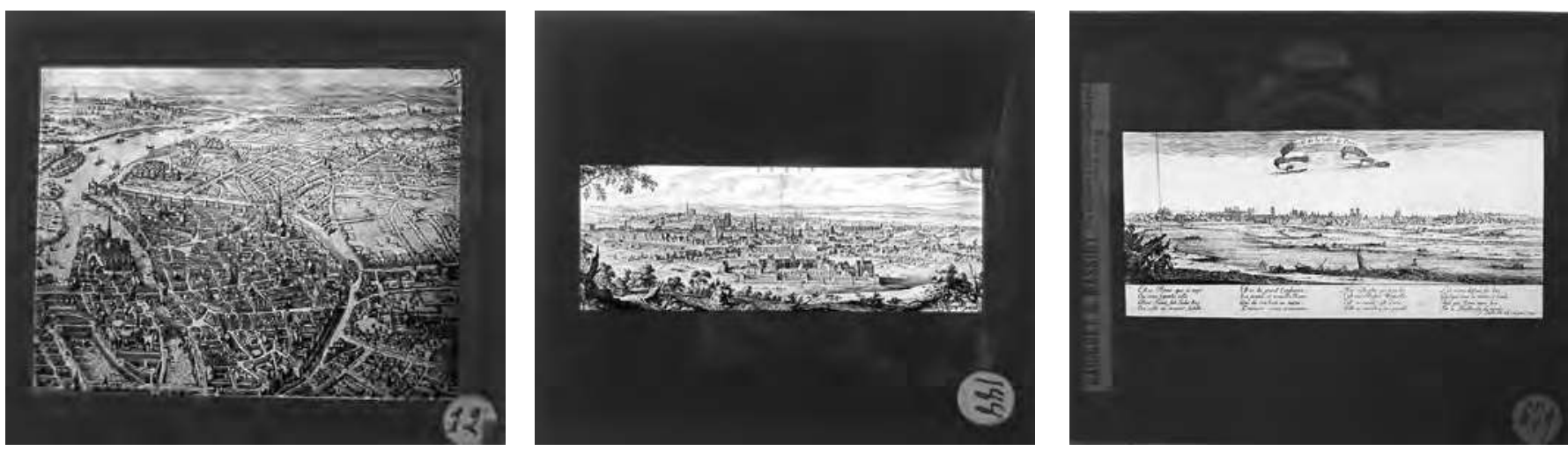

\section{Livret de présentation de l'exposition «Paris durant la Grande Époque Classique » (1911) \\ Extrait 1 : « L'exposition »}

Qu'était Paris durant la Grande Époque Classique formée du XVII siècle ? Telle est la question à laquelle la présente Exposition essaye, dans son cadre limité de répondre. Le visiteur voudra bien suivre, autant que possible, l'ordre des vitrines. II verra ainsi plus commodément se dérouler ce modeste essai de synthèse ou de leçon de choses. Après avoir pris contact avec la ville à vol d'oiseau, tel un voyageur, qui, se rendant à Paris, peut en embrasser à quelque distance le panorama - il parcourra les approches de cette grande cité envisagée dans les limites du tracé des boulevards ou du cours de Louis XIV, tant sur la rive gauche que sur la rive droite. II reconnaîtra les voies principales servant d'accès à la capitale ou par lesquelles elle s'alimente; il observera les lignes essentielles de son sol. La grande artère de vie de Paris, la Seine, le fera pénétrer dans cette ville dont toute la partie bordant le fleuve pourra lui devenir familière. II lui sera loisible du Pont-Neuf et le centre de vie populaire que constituent ce pont et ses abords. À ce centre s'opposera le centre de vie mondaine du Marais. Puis le Louvre et les Tuileries ne lui feront pas quitter cette vie élégante, tout en lui permettant de rattacher particulièrement à ces lieux une part essentielle de la gloire du Xv/l siècle parisien. Le voici dans ces parages de l'ouest de la rive droite jalonnés de ces monuments : le Palais-Royal, la place des Victoires, la place Vendôme. II n'abandonnera point toute cette partie de Paris sans assister au fameux carrousel de 1662. De la Cour et de ce qui s'y rattache, il passera à la Ville, personnifiée par l'Hôtel de Ville. Une promenade à travers la rive gauche lui laisse encore à visiter l'île de la Cité, pleine d'une animation diverse et qui marque un point urbain à peu près central par rapport à la promenade des boulevards ou du cours terminant les vitrines topographiques ou de pourtour. Le centre de la salle sert à présenter la vie parisienne dans ce cadre topographique : De la naissance à la mort, l'Exercice des métiers, la Rue, I'Eau, les Heures du jour, Paris la nuit, la Cour des Miracles, la Vie des Halles, la Fronde, Hôtels et Jardins, le Théâtre, Port-Royal, tels sont les principaux ensembles soumis à son bienveillant examen, sans parler de pièces encadrées ou groupées à part dans deux grandes vitrines plates.

Des imprimés jugés significatifs voisinent avec des gravures pour un mutuel éclaircissement. Des plans sont présentés, en vertu du même principe de méthode, dans l'escorte d'estampes susceptibles d'en devenir ainsi comme le commentaire figuré. Les gravures elles-mêmes se trouvent conjointes pour permettre, indépendamment de la simple vision des lieux figurés, des rapprochements de critique iconographique.

Quant aux vieux artistes qui ont laissé l'image du grand Paris classique qu'ils ont connu, ils se livrent à vous avec leur manière d'art et leur valeur documentaire. Vous les présenter successivement et à ce double point de vue serait dépasser les proportions de cette Notice. 

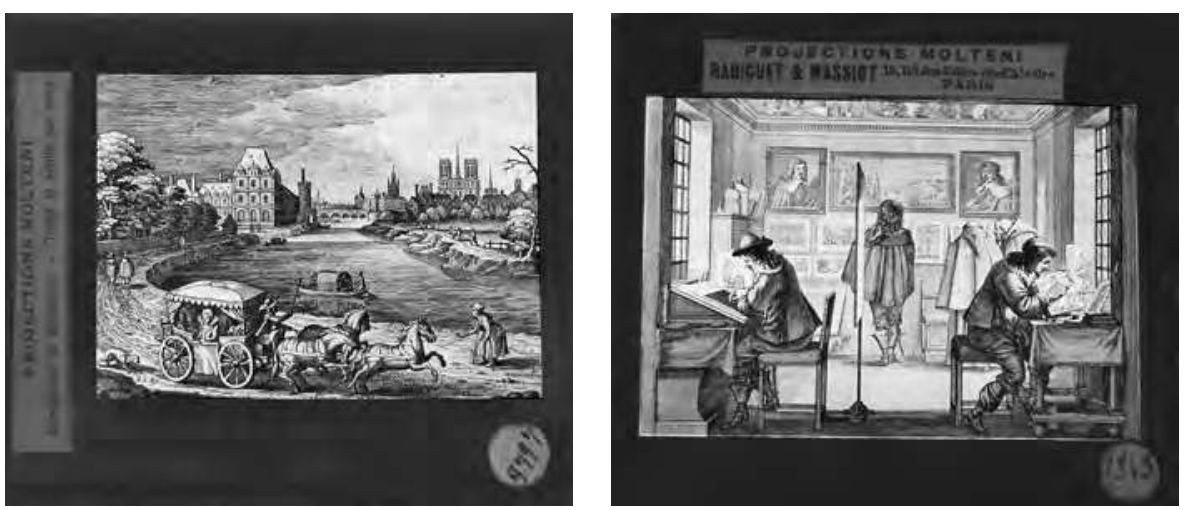

Plan de Paris (extrait). Eau-forte, Matthäus Merian, vers 1615.

Vue de Paris en surplomb. Eau-forte, Matthäus Mérian, vers 1615.

"Profil de Paris ». Eau-forte, Israël Sylvestre,

« Paris ». Eau-forte, Matthäus Mérian, vers 1615 .

«L'atelier du graveur ".

Eau-forte, Abraham Bosse, 1624.

\section{Extrait 2 de la section}

\section{« En route pour Paris. La ville à vol d'oiseau "}

Dans ces vitrines se succèdent des gravures comme celles que regardent avec attention le gentilhomme et les deux capucins qu'Abraham Bosse, en une estampe datée de 1643 , nous montre en visite dans un atelier de graveurs. De même que le voyageur que cet artiste nous représente [...] dirigez-vous vers la " grand' ville » que semble vous désigner du doigt un personnage accompagné d'une grande dame ou que vous montre un groupe de cavaliers à la romaine. Arrivé à un endroit d'où l'on peut embrasser du regard le panorama de Paris, arrêtez-vous, à la manière de cet autre groupe que nous présente (1649) le graveur florentin Stefano della Bella. Et même il vous sera loisible de faire comme ce dernier, de vous asseoir et de dessiner un croquis du paysage qui se déroule devant vous, au temps où s'ouvre le règne de Louis XIV. Ou bien, avec Matthaüs Merian, de Bâle, vous remonterez de quelques années et considérerez, sous le double aspect d'un plan cavalier et d'une vue panoramique, le Paris de la fin du règne de Henri IV et des premières années du règne de Louis XIII.

Sur la rive droite, en avancée au-delà de la ligne du rempart, I'Hôpital Saint-Louis, création de Henri IV, prend les proportions d'une petite cité isolée. II est séparé du rempart par des cultures maraîchères, "les marets du Temple », qu'un ruisseau d'égout traverse, se dirigeant vers « la grande Batellière », pour, de là, se déverser dans la Seine, à l'endroit de notre place de I'Alma. Puis, voici le rempart, précédé d'un fossé et où se succèdent des bastions que surmontent des groupes de moulins à vent. Ce rempart, qui fut commencé au $X V I^{e}$ siècle et poursuivi sous Louis XIII, a étendu vers l'ouest, par rapport à la précédente enceinte encore visible de ce côté, le Paris de la rive droite, englobant notamment dans la ville la butte Saint-Roch coiffée de moulins. II a ainsi réalisé à peu près le tracé de nos grands boulevards actuels, tandis que la rive gauche reste avec sa vieille enceinte de PhilippeAuguste comprise approximativement entre ces trois points du Paris de nos jours : I'Institut, le Panthéon, la rue du Cardinal-Lemoine.

De la fin environ du XVII siècle date la vue panoramique située au-dessous de celle de Mérian. Elle est prise de la rive droite, dont la succession des faubourgs se déroule depuis l'abbaye de Saint-Antoine et le «Thrône » jusqu'au faubourg Saint-Denis. L'animation du premier plan nous montre des cavaliers, des chasseurs, des voitures diverses, des ânes pesamment chargés, voire même un pèlerin. Des terres de culture, des jardins coupés de murs, avec des maisons égrenées çà et là, occupent le sol jusqu'au pied du rempart que surmontent les lignes d'arbres des nouveaux boulevards datant de 1670. Dans les fonds, les villages de la banlieue laissent apparaître leurs humbles clochers, et le paysage se complète par de nombreux moulins à vent. Au-dessus de la ville entassée, tours et flèches émergent à côté des dômes, l'une des caractéristiques de l'architecture du siècle et qui marquent l'emplacement d'édifices tels que le Val de Grâce, la Sorbonne, 

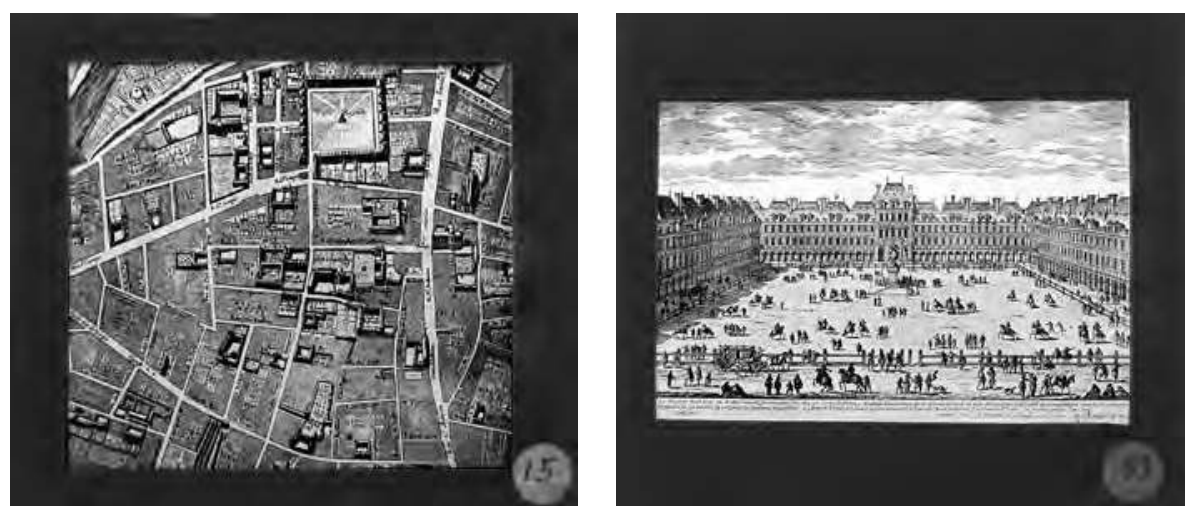

Plan de Gomboust. Eau-forte, Jacques Gomboust, milieu du xVII siècle.

Place royale. Eau-forte, Gabriel Perelle, milieu du xvII siècle.

les Jésuites de la rue Saint-Antoine, les Invalides, les Filles de l'Assomption. Autre note nouvelle dans le paysage parisien : c'est l'Observatoire.

De la fin également du règne de Louis XIV est cette "Vue de Paris du côté du nord ", comprise, au premier plan, entre la hauteur de Belleville et celle de Montmartre, avec le vaste enclos de Saint-Lazare, "la Guinguette ", les Porcherons. Vous y observerez, ces grandes constructions du XVII siècle : la Salpétrière et l'Arc de triomphe du faubourg Saint-Antoine.

C'est au contraire une vue de Seine inspirée d'un " profil de la ville de Paris » d'Israël Silvestre et nous laissant voir la ville de la rive gauche en aval des Tuileries, que semblent nous présenter deux personnages contemporains de Louis XIV, tandis qu'à côté, Aveline nous montre Paris pris du faubourg Saint-Marcel.

À cheval, en coche ou en carrosse, vous êtes donc parvenu près de Paris, dont la porte de la Conférence, à l'extrémité sud-ouest du jardin des Tuileries, s'offre devant vous, par exemple, comme elle apparaît au bœuf des fables de La Motte s'acheminant vers la ville. Sans doute rencontrez-vous tel ou tel Lombard ou Savoyard venant à Paris pour y être ramoneur, tel ou tel Limousin désireux d'y exercer le métier de maçon, à moins que ce ne soit un de ces Espagnols qui, durant la première moitié environ $d u x \|^{e}$ siècle, se trouvaient nombreux dans la capitale.

\section{Conférence à la BHVP (1910) Extrait}

Dans une première conférence sur la Place Royale, j'ai essayé de vous montrer comment cette place a été formée ; dans la conférence suivante, c'est l'utilisation de la place, une fois formée, qui nous a retenus. Aujourd'hui, je voudrais vous parler de la vie à la Place Royale. Les espaces libres qui existent dans la ville, sous la forme de place ou de promenoir (comme on disait alors) ont en effet le privilège de refléter certains aspects de la vie de cette ville : ils peuvent constituer, à cet égard, comme un miroir.

Enfin, et c'est également un point de vue qui nous occupera aujourd'hui à propos de la Place Royale, l'espace libre constitué, autrement dit la place et la promenade, agit comme élément de peuplement ou de transformation urbain dans la partie de la ville où il se trouve : cet espace libre, place ou promenade, se trouve exercer une sorte d'action réflexe, destructrice de l'espace libre environnant.

C'est ce qui s'est produit pour la Place Royale. Une fois créée, elle a attiré de son côté une part de la vie parisienne et, du même coup, a contribué au développement urbain et au peuplement des lieux voisins jusque-là dépourvu d'habitations et utilisés surtout comme cultures maraîchères.

La place royale a engendré la vie urbaine de ce qu'on appelle le Marais... 


\section{$L^{\prime}$ « art de ville » à sa racine}

Ce serait, affirme Poëte, dès la fin du $\mathrm{xv}^{\mathrm{e}}$ siècle qu'apparaissent quelques premières figurations gravées dans lesquelles le spectateur du $x^{e}$ siècle reconnaît les lieux de Paris dans lesquels il évolue. Une centaine d'années plus tard, à la suite d'une modernisation des méthodes d'impression, il y aura une intensification de la production et de la distribution de vues gravées présentant l'image d'une ville qui se transforme. Réunies sous forme d'albums (ou parfois d'atlas), elles seraient, encore selon Poëte, à la fois le véhicule et l'indice d'une forme de prise de conscience de la ville dans sa totalité, et, dit-il, dans sa nouvelle beauté qui s'incarne à travers le dégagement des grandes espaces de mouvement et des jardins aux tracés en ligne droite. En effet, pratiquement l'intégralité des premières 600 images acquises sous forme de projections photographiées (ceci au moment de l'exposition sur la vie populaire, qui n'a présentée que des documents originaux) datent de cette époque. De plus, dans les années qui suivront et jusqu'en 1913, la collection n'arrêtera pas d'en intégrer d'autres: ayant épuisé, semble-t-il, les réserves de telles vues dans les collections publiques, I'historien continuera à en acquérir en faisant appel aux collectionneurs qui, auparavant, avaient nourri de pièces originales les deux expositions précédentes de la BHVP.

Dans un sens, le fait que le Paris de l'époque classique soit la mieux représentée dans la collection iconogra- phique ne saurait surprendre. C'est la période sur laquelle l'historien reviendra le plus souvent par la suite et, on serait tenté de dire, que c'est celle dans laquelle I'archive iconographique trouve son origine, voire sa raison d'être.

L'idée même d'un moyen de représentation et de connaissance " objectif ${ }^{1}$ " du réel apparaît, nous rappelle-til, avec de telles vues gravées: en même temps, et comme par la même impulsion, apparaît, toujours selon lui, l'« art de la ville ".

Il est plus que probable que l'idée même d'une collection iconographique vient de là. En effet, le regroupement effectué sous la direction du bibliothécaire en chef de la Bibliothèque historique dès 1907 ne serait presque qu'une reprise de la partie parisienne des collections d'estampes qui se trouvaient dans les collections royales de Versailles sous Louis XIV. Et il se trouve qu'Israël Sylvestre (artiste dont l'intégralité ou presque des 200 vues de Paris se trouve dans la Collection iconographique de la BHVP), a été « Dessinateur ordinaire du roi » en plus d'être collectionneur de vues gravées des villes et des édifices. Par ailleurs, Gabriel Perelle, conservateur des cartes et plans du Cabinet du roi, est également auteur de nombre de vues parisiennes figurant aussi dans la collection.

Aux vues gravées ou dessinées de Perelle et de Sylvestre se rajoutent, entre autres, celles de Bosse, de Mérian, de Braun, de Callot et de Bella (dont on voit ici des exemples). Logiquement, leurs représentations de
Paris reflètent, de façon différente, une stricte codification des conventions qui, dans l'art classique régissent l'imitation du réel: à commencer par celle de la représentation du sujet ou groupes de sujets qu'ils montrent ${ }^{2}$.

Or ce sujet est, très souvent, promeneur ou promeneuse. La promenade serait alors une pratique qui se répand dans les nouveaux espaces dégagés de la ville, et dans ses environs: elle se pratique sous une diversité de formes, plus ou moins luxueuses ou populaires, toujours suivant des codes sociaux définis. Cependant, en ce qui concerne les vues qui nous intéressent, le promeneur est également le personnage qui représente le parcours spatial que le spectateur est invité à retracer, de l'œil, à travers chaque vue et d'une vue à une autre. Son positionnement est variable d'image en image. Il peut être situé devant un espace à parcourir, être montré en train de traverser une vue en perspective, assis au repos pour parcourir de l'œil le "profil " d'une ville ou une vue d'ensemble en surplomb. Il est à noter que le promeneur en question est souvent l'artiste lui-même (voir la vue panoramique de Braun).

Ainsi, de vue en vue, par la promenade imaginée, se construit l'image de l'ensemble de la ville, à travers différents genres de vues qui se relaient pour construire une représentation totale de la ville: le plan " cavalier ", les " profils " (ancêtres des panoramas du XIX siècle) ou des vues depuis des hauteurs dans les environs de la ville; des vues de promeneurs dans les
1. Le terme " objectif » est utilisé ici selon le sens que Philippe Descola lui attribue dans l'ouvrage qu'il a dirigé, La Fabrique des images : visions du monde et formes de la représentation, Paris, Somogy, 2010. Voir en particulier le chapitre intitulé « Un monde objectif ». L'anthropologue évoque l'apparition « d'un point de vue naturaliste qui commence à émerger en Europe au xvII siècle » (p. 73), menant à la représentation des « contiguités matérielles [des personnes figurées] au sein du monde physique qui gagne son indépendance ». Descola évoque également la façon dont « l'autonomisation et la sécularisation de l'espace conduisent.à la naissance d'un autre genre [picturale], le paysage », (p. 80).
2. Nous devons notre approche de ces œuvres à Louis Marin. Voir « La ville dans sa carte et dans son portrait », De la représentation, Paris, Gallimard-Le Seuil, coll. « Hautes études », 1994. 
champs entourent la ville, souvent sur des routes qui conduisent à ses portes; des vues en perspective des cours, rues, ponts ou autres pièces urbaines; des scènes présentant des paysages urbains proches ou lointains à vue d'œil ou en surplomb et remplis de personnages et d'objets; des vues en perspective, " cavalières ", ou en plan et en façade d'architectures; des représentations plus ou moins réalistes ou allégoriques des métiers de rue, des saisons, des vertus etc; des scènes présentant des conditions de vie, dont par exemple la vue (reproduite ici) illustrant « Les embarras de Paris " de Nicolas Boileau; enfin, des portraits de promeneurs vus de près, entourés des quelques détails du cadre dans lequel ils se trouvent. Avec les cours de 1910-1911 sur l'époque classique (dont un court extrait est publié ici) et l'exposition de 1911, se fixent les prémisses d'une conception de l'art de ville, du moins tel qu'il peut en exister à Paris, que I'historien ne fera que développer ou redire par la suite. Dès 1919, I'année où Poëte et ses proches créent l'Institut d'histoire, de géographie et d'économie urbaines, il commencera une nouvelle collection - cette fois de pièces originales d'estampes et d'imprimés de villes autres que Paris. Bon nombre des quelques 1500 pièces acquises à terme ${ }^{3}$ seront des cartes et des plans d'autres villes européennes datant de l'époque classique, offrant des conditions de visualisation similaires à celles de Paris qui se trouvaient déjà dans sa collection photographiée.

S. M.

3. Autant « inconnue » que les clichés de projection, cette collection de la BHVP n'est pas officiellement repérée en tant que partie des ressources de la bibliothèque, et n'est pas communiquée.
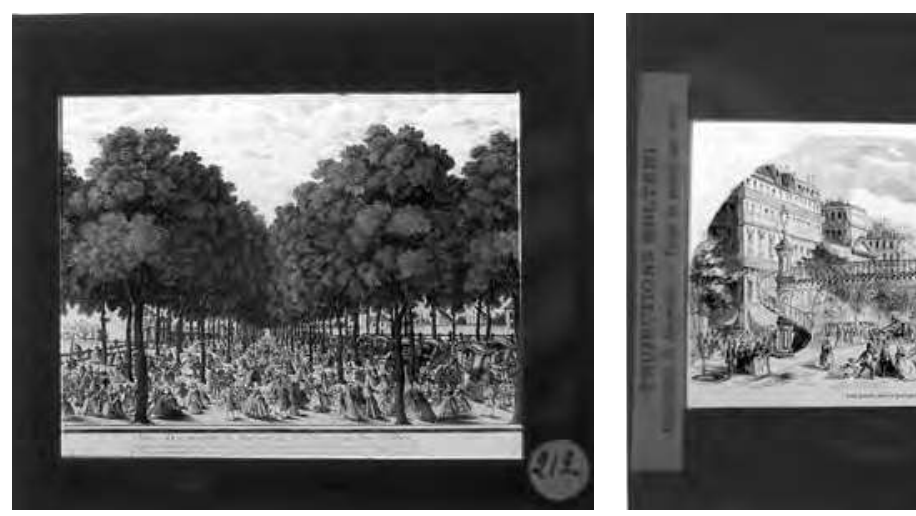

Sur les boulevards. Madeleine-Bastille. (Depuis le XVII siècle jusqu'à la fin du Second Empire.)

\section{Extrait}

Omnibus, coucous, citadines, tilburys, véhicules divers encombrent la chaussée. Les trottoirs, envahis par les cafés, deviennent trop étroits. La traversée des boulevards est un difficile et périlleux exercice, auquel ne se hasardent qu'avec crainte le bourgeois timide et la promeneuse effarouchée. Pour obvier à ce sérieux inconvénient, des projets divers voient le jour : passerelle légère jetée d'un côté à l'autre (1857) ; passage souterrain "à l'instar de ceux de Londres " (1862). En 1865, le premier "refuge » est créé sur le boulevard des Capucines, au carrefour des rues de Sèze et Caumartin, et cette innovation montre bien le mouvement de circulation qui s'est, depuis la création de l'Opéra, porté vers cette partie du boulevard. II n'est pas sans intérêt de relever en outre l'existence d'un projet de chemin de fer souterrain, dont des pièces figurent deux coupes aux boulevards Bonne-Nouvelle et des Capucines.

Le Second Empire a vu le triomphe du macadam, dont on avait, sous Louis-Philippe, commencé à doter les boulevards. Le public cependant ne s'était pas fait faute de railler tout d'abord l'invention nouvelle... 

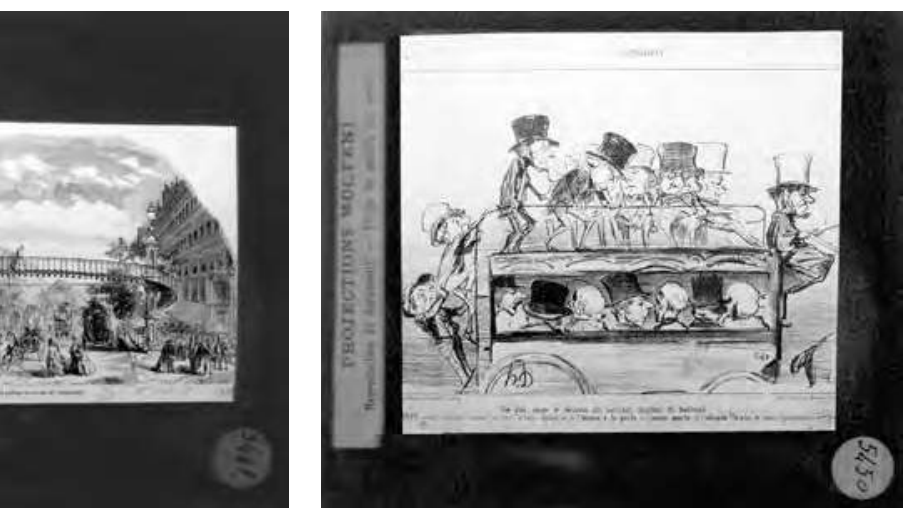

Cours Saint-Antoine.

Lithographie, Celestin Deshaye, autour de 1835.

«Pont projeté pour le passage des piétons au-dessus des boulevards ". Lithographie, 1887.

"Vue plan coupé et élévation des nouveaux omnibus du boulevard ". Lithographie, Honoré Daumier, 1853.

(C) Steven Melemis

Promenades et transformations urbaines

Revenant sur des éléments déjà présentés dans l'ensemble des expositions précédentes, celles de 1912, « Sur les boulevards ${ }^{1}$ ", comme celle de 1913, "Promenades et jardins de Paris ${ }^{2}$ " proposent des évocations particulièrement denses de la "physionomie " de ces deux composants à partir desquels des approches de l'extension urbaine pourront être conçues. Ensemble, elles résument la formation et les évolutions à travers le temps des formes urbaines jusqu'à ce qu'elles assument l'état dans lequel elles seront au début du $\mathrm{xx}^{\mathrm{e}}$ siècle. Dans I'une comme dans l'autre, la polysémie associée à la promenade est manifeste. Revisitant l'ensemble des thématiques des expositions précédentes, elles " généralisent ${ }^{3}$ " le thème de la promenade de façon à ce qu'elle puisse informer les réflexions sur les transformations urbaines à venir. S'il n'y a pas de grande surprise au niveau des types de pièces graphiques, photogra- phiques ou textuelles déjà exposées, grâce à de nombreuses contributions nouvelles des collectionneurs de la Société d'iconographie parisienne, elles rajoutent une épaisseur supplémentaire à l'évocation du quotidien des lieux de vie en question. Les temporalités qui rythment la journée ressortent de manière plus nette. Une abondance de détails de modifications mineures et parfois éphémères révèlent des réseaux de relations qui ne cessent de mettre en correspondance active la « vie urbaine " avec " son " cadre: la taille des arbres de façon à dégager une vue de la rue; I'implantation d'éclairages; l'édification de kiosques et autres petites constructions; le fonctionnement au quotidien des omnibus; les modifications temporaires au niveau des circulations et les effets globaux qu'elles provoquent, etc. Parfois insignifiantes ou peu importantes en soi, ces observations de détail offrent une sorte de "leçon de choses " par rapport à cette écologie que l'auteur appelle la « vie urbaine ».

L'« Aperçu historique ", premier volume du Rapport à la Commission d'extension de Paris, opère, autour des projets d'extensions du passé parisien, un troisième retour thématique sur les expositions des années précédentes. Cependant, dans sa version définitive, les évocations " vivantes " que son auteur affectionne disparaissent ${ }^{4}$. Copieuses, les illustrations intégrées au rapport ne servent qu'à illustrer le texte de plans et de projets. Les pressions exercées par les populations urbaines n'y sont évoquées que par leurs effets sur la conception ou la réalisation des projets. D’une remarquable précision et clarté, cet "Aperçu » historique faisant partie d'un rapport d'urbanisme est l'une des rares publications de Marcel Poëte à être bien reçue par ses collègues historiens ${ }^{5}$.

S. M.
1. L'intitulé complet est : «Sur les boulevards Madeleine-Bastille (Depuis le XVII siècle jusqu'à la fin du Second Empire ».

2. L'intitulé complet est : «Promenades et jardins de Paris (Depuis le Xve siècle jusqu'en 1830)».
3. Comme la présentation de 1907 l'avait fait pour la notion de vie populaire.

4. Il existe une étape du manuscrit dans les Papiers Poëte qui présente quelques différences intéressantes avec la version finale du texte de l'Apogée historique, BHVP, MS, 191.
5. Lucien Febvre en parlera très favorablement dans son compte rendu pour la « Revue historique » de 1930 (assez peu enthousiaste) de l'Introduction à l'urbanisme. 


\section{Dans la polysémie de la "leçon de choses"}

Les expositions de la période de 1907 à 1913 de la BHVP seraient autant de "leçons de choses "; c'est le terme que Marcel Poëte emploie dans plusieurs livrets. Il signifie, entre autres, une forme d'enseignement qui part d'un objet concret, ou bien d'une image d'un tel objet, pour accéder à une compréhension d'un phénomène ou une idée plus abstraite, en s'appuyant sur la connaissance pratique du monde de l'apprenant. La notion avait servi comme fondement de l'éducation publique en France depuis 1870, à commencer par les enseignements de l'école primaire pour atteindre, vers la fin du siècle, le niveau du lycée et de l'université ${ }^{1}$. Dans les expositions qui nous intéressent, on pourrait dire qu'elles évoquent une mobilisation de la connaissance pratique du spectateur - a priori un habitant ou habitué de Paris - dans la reconstitution du passé de la ville voire dans l'imagination de possibles futurs pour elle. Les " choses" en question, dans le cadre des expositions, ce sont les vues dessinées: ces documents, grâce au regard critique et au prix d'un effort d'imagination, qui permettront au spectateur de saisir des renseignements sur les états " historiques " d'un espace urbain donné, en mobilisant son expérience personnelle de l'espace en question. Pour opérer au mieux cette saisie, I'œil du spectateur est invité à parcourir non pas une image, mais plusieurs.
Le " parcours » de plusieurs vues I'amènera à s'imaginer parcourir réellement l'espace représenté par les images. En effet, c'est grâce au mouvement, à l'action pratique et à la perception, ou plutôt à l'imagination de ces trois éléments, qu'il pourra construire de la connaissance à travers l'image, faisant écho à l'acquisition de connaissance acquises grâce à ses " promenades " à travers des lieux réels. En effet, c'est dans l'effort de comparaison d'une série d'états historiques des espaces de la ville avec sa connaissance des espaces réels dans le présent que le spectateur pourra entr'apercevoir ce que l'historien appelle, dans ces années à cheval entre une époque passée de transformation et une autre imminente : la « vie urbaine ".

S. M.

Remerciements

Nous tenons à remercier mesdames Emmanuelle Toulet, directrice de la Bibliothèque historique de la Ville de Paris, Juliette Jestaz, adjointe à la directrice, Carole Gascard, responsable des collections photographiques, pour leur aide indispensable lors des explorations des archives non communiquées, qui ont rendu possible cette recherche. Nous remercions également et chaleureusement, monsieur Francis Perey, petit-fils de Marcel Poëte, de nous avoir accordé le droit de publier des extraits de textes et de notes figurant dans les Papiers Poëte.

1. Voir Ferdinand Buisson, « Leçon

de choses », entrée dans le Nouveau

Dictionnaire de pédagogie et d'instruction primaire, Hachette, 1911, du même auteur, 1911. 